\title{
FIELD IONIZATION OF SHALLOW ACCEPTORS
}

\author{
A. Dargys, S. Žurauskas and N. Žurauskienė \\ Semiconductor Physics Institute, A. Goštauto 11, 2600 Vilnius, Lithuania \\ - Experimental studies on hole tunneling from the substitutional boron \\ impurity into degenerate valence band of silicon single crystals are presented. \\ The results are interpreted within the framework of acceptor ground state \\ quartet splitting into the Kramers doublet due to presence of random strains \\ and electric field in the lattice.
}

PACS numbers: $71.70 . \mathrm{Ej}, 73.20 . \mathrm{Hb}, 79.70 .+\mathrm{q}$

\section{Introduction}

Acceptor field ionization in semiconductors having degenerate valence band edge has been considered theoretically in [1], where it has been concluded that the light holes basically will dominate the tunneling. However, in real semiconductors, due to the presence of dislocations, residual and intentionally introduced impurities, the valence band edge and acceptor level degeneracies are partially lifted. Also, as shown in [2], the acceptor tunneling dynamics may be influenced by the acceptor Stark effect [3]. Thus, the experimental investigations are needed to get the true picture of the acceptor-to-valence-band tunneling. Below we present the first preliminary results on acceptor field ionization dynamics. Single crystals of silicon doped by boron impurity have been investigated.

\section{Experiment}

Acceptor-to-valence-band tunneling dynamics was measured with the help of transient tunneling spectroscopy (TTS), a kind of nonresonant spectroscopic technique [4]. In TTS a fast linearly increasing voltage is applied to a plate-shaped sample with blocking contacts on one or two opposite faces, and induced transient current flowing through the sample is monitored as a function of time.

The experiments were performed on boron doped commercial silicon having room temperature resistivities 10 and $40 \Omega \mathrm{cm}$. The lightly-doped samples have been used to avoid impurity impact ionization by hot carriers at high electric fields [5]. The $n^{+}$- and $p^{+}$-contacts were formed on opposite faces of samples by diffusing phosphorus and boron to the depth of a few micrometers. The metal electrodes were obtained by the final evaporation of $\mathrm{Cr}-\mathrm{Pd}-\mathrm{V}-\mathrm{Cu}$. The useful $p$-region length and area of resulting $n^{+}-p-p^{+}$samples was $d=100 \mu \mathrm{m}$ and 
$S=1 \mathrm{~mm}^{2}$, respectively. At the investigated temperatures all free holes are frozen out on boron atoms, therefore, at low reverse voltages applied between $n^{+}$- and $p^{+}$-contacts the samples behaved as small, $1 \mathrm{pF}$ capacity condensers.

In Fig. 1a there is shown time dependence of the voltage over the $40 \Omega \mathrm{cm}$ sample and in Fig. 1b the corresponding current spectrum at some fixed lattice temperatures. Sudden increase in the current at $t \approx 17 \mathrm{~ns}$ is associated with the

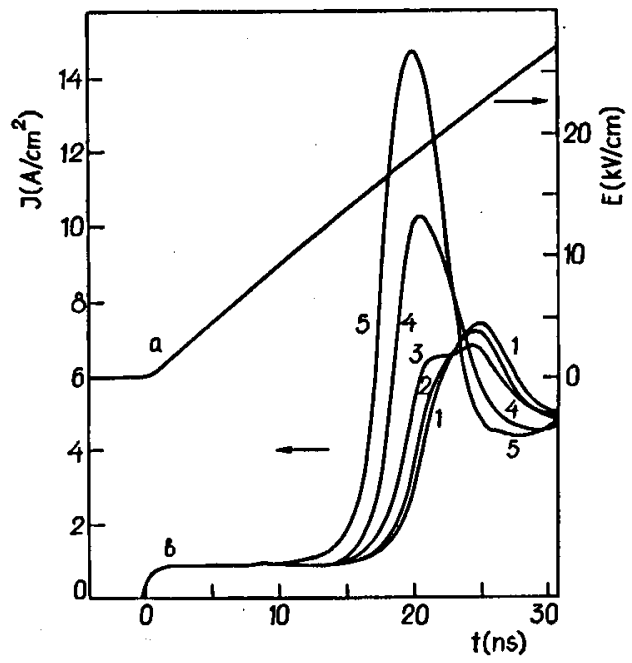

Fig. 1. Time dependence of the voltage over and transient current through the $40 \Omega \mathrm{cm}$ $p$-Si〈B $\rangle$ sample at lattice temperatures: $1-4.2 \mathrm{~K}, 2-10 \mathrm{~K}, 3-15 \mathrm{~K}, 4-17 \mathrm{~K}$, $5-20 \mathrm{~K}$.

fast switching of the acceptor-to-valence-band tunneling transitions, while the decrease in the tunnel current at the later moments is associated with (i) exhaustion of neutral boron atoms capable to supply holes to valence band and (ii) formation of space-charge region near $n^{+} p$-contact. At low temperatures the tunneling pulse is double-humped, however, with the increase in lattice temperature the pulse transforms into a single-humped one. The double-to-single pulse transformation was observed in all investigated samples, although, the detailed structure of the double-humped pulse, sometimes rather poorly resolvable, has changed from sample to sample.

\section{Discussion}

The observed double-to-single pulse transformation cannot be explained by hole tunneling from a single acceptor energy level. We think that the observed tunneling current spectra transformation is associated with a splitting of the boron ground level by random lattice strains and/or by electric field (acceptor Stark effect).

As known, the fourfold degenerate $\Gamma_{8}$-ground level of boron atom under the influence of elastic strains or electric fields of lower than cubic symmetry will be 
split into the Kramers doublet characterized by projection of the total angular momentum of the hole: $M_{J}= \pm 1 / 2$ and $\pm 3 / 2$. The presence of the random internal strains is essential in explaining the ultrasound attenuation in lightly doped $p$-Si at low temperatures [6] and the absence of shallow acceptor paramagnetic resonance [7]. The edge of the valence band has exactly the same $\Gamma_{8}$-symmetry, therefore, the valence band edge will suffer similar splittings in the presence of the fields. The splitting, as shown in [2], may have profound influence on the acceptor-to-valence-band tunneling dynamics. Due to selection rules, the hole tunneling transitions from the particular split level will be allowed to either light or heavy mass subband, or, depending on the direction of the field, to both. The tunneling time from the discrete level to a continuum, in general, can be approximated by

$$
\tau_{t}=\Omega \exp \left(\frac{4}{3} \frac{\sqrt{2 m}}{e \hbar} \frac{\varepsilon_{\mathrm{i}}^{3 / 2}}{E}\right) \equiv \Omega \exp (\alpha / E),
$$

where $m=m_{\mathrm{h}}$ or $m_{\mathrm{l}}$, depending on the band to which the hole tunnels, is the heavy or light mass of the hole. $\varepsilon_{\mathrm{i}}$ is the ionization energy of the level and $E$ is the electric field. The pre-exponential factor $\Omega$ depends on the form of the acceptor potential. Figure 2 illustrates the calculated tunneling current spectrum when in

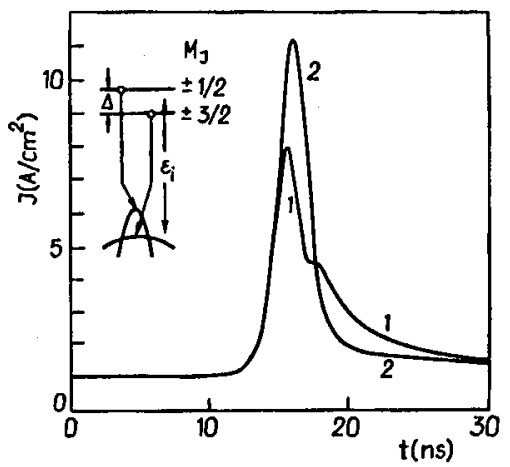

Fig. 2. Calculated transient currents for the two values of the Raman scattering time between the split ground state levels: $\tau_{\mathrm{R}}=15 \mathrm{~ns}$ (curve 1) and $\tau_{\mathrm{R}}=0.15 \mathrm{~ns}$ (curve 2). $N_{ \pm 1 / 2}=N_{ \pm 3 / 2}=2.5 \times 10^{13} \mathrm{~cm}^{-3}, \mathrm{~d} U / \mathrm{d} t=10^{10} \mathrm{~V} / \mathrm{s}$. The insert shows the tunneling transitions from the split acceptor ground state to heavy and light mass bands, respectively. $\Delta \ll \varepsilon_{\mathrm{i}}$.

the tunneling dynamics both the acceptor-to-heavy-mass-subband transition and acceptor-to-light-mass-subband transition are included through the parameter $\alpha$ in (1): $\alpha_{\mathrm{h}}=515 \mathrm{kV} / \mathrm{cm}$ and $\alpha_{1}=258 \mathrm{kV} / \mathrm{cm}$. The pre-exponential factor in (1) was approximated by the formula $\Omega=E / \omega \alpha$, which is exact for the ground state of the scaled hydrogenic impurity atom: $\omega=12 \varepsilon_{\mathrm{i}} / \hbar=8.4 \times 10^{14} \mathrm{~s}^{-1}$; $\varepsilon_{\mathrm{i}}=45.71 \mathrm{meV}$ for the boron impurity in silicon. Two curves in Fig. 2 correspond to two different Raman scattering times of acoustic phonons, $\tau_{\mathrm{R}}$, by boron atoms. The Raman scattering tends to thermalize the hole population within the 
Kramers doublet characterized by $M_{J}= \pm 1 / 2$ and $\pm 3 / 2$ during the voltage ramp. In Fig. 2 the values $\tau_{\mathrm{R}}=15 \mathrm{~ns}$ and $0.15 \mathrm{~ns}$ approximately correspond to lattice temperatures 4.2 and $18.5 \mathrm{~K}$, respectively [6]. Figure 2 clearly demonstrates that in the case of low thermal coupling between ground state Kramers levels (curve 1) the spectrum is double-humped while in the case of strong coupling (curve 2) the spectrum transforms into single-humped one. In the latter case the holes tunnel out of the acceptors exclusively through the band characterized by light mass in the direction of the field. Here it should be noted that in the calculation the acceptor-to-valence-band tunneling in all cases has been considered to be elastic, i.e. without phonon participation and that in the tunneling dynamics the effective masses rather than energy splitting $\left(\Delta / \varepsilon_{\mathrm{i}} \approx 10^{-4}\right)$ play the main role. Finally, it will be remarked that in the simulation the space charge effects were included fully.

In conclusion, the preliminary experimental results show that acceptor ground state quartet splitting into the Kramers doublet and ensuing spin selection rules may have profound influence on the acceptor-to-valence-band tunneling dynamics in common $p$-type semiconductors.

\section{References}

[1] Hui-Quan Nie, D.D. Coon, Solid State Electron. 27, 53 (1984).

[2] A. Dargys, submitted to press.

[3] G.L. Bir, E.I. Butikov, G.E. Pikus, J. Phys. Chem. Solids 24, 1467, 1475 (1963).

[4] A. Dargys, S. Žurauskas, N. Z̆urauskienè, Appl. Phys. A 52, 13 (1991).

[5] A. Dargys, S. Žurauskas, Solid State Commun. 52, 139 (1984).

[6] T. Ishiguro, Phys. Rev. B 8, 629 (1973).

[7] G. Feher, J.C. Hensel, E.A. Gere, Phys. Rev. Lett. 5, 309 (1960). 